\title{
How Myalgic encephalomyelitis / Chronic fatigue syndrome (ME/CFS) progresses: A framework for research and the prevention, treatment, and rehabilitation in ME/CFS
}

Luis Nacul ${ }^{1,2}$, Shennae O’Boyle ${ }^{1 *}$, Flavio E. Nacul ${ }^{3}$, Kathleen Mudie ${ }^{1}$, Caroline C. Kingdon ${ }^{1}$ Jacqueline M Cliff $^{1}$, Taane G. Clark ${ }^{1,4}$, Hazel M. Dockrell ${ }^{1}$ and Eliana M. Lacerda ${ }^{1}$

${ }^{1}$ Faculty of Infectious and Tropical Diseases, London School of Hygiene \& Tropical Medicine, London, United Kingdom

${ }^{2}$ B.C. Women's Hospital \& Health Centre, Vancouver, Canada

${ }^{3}$ Pro-Cardiaco Hospital and Federal University of Rio de Janeiro, Rio de Janeiro, Brazil

${ }^{4}$ Faculty of Epidemiology and Population Health, London School of Hygiene \& Tropical Medicine, London, United Kingdom

*Corresponding Author: Shennae.OBoyle@1shtm.ac.uk 


\begin{abstract}
We propose a framework for the understanding of the pathophysiology and management of Myalgic Encephalomyelitis / Chronic Fatigue Syndrome (ME/CFS) that considers wider determinants of health and long-term temporal variation in pathophysiological features and disease phenotype throughout the natural history of the disease.

As in other chronic diseases, ME/CFS evolves through different stages, from asymptomatic predisposition, progressing to a prodromal stage, and then to symptomatic disease. Disease incidence depends on genetic makeup and environment factors, the exposure to an insult, or repeated insults, and the nature of the host response. In people who develop ME/CFS, normal homeostatic processes in response to adverse insults may be replaced by aberrant responses leading to dysfunctional states. Thus, the predominantly neuro-immune and autonomic manifestations, underlined by a hyper-metabolic state, that characterise early disease, may be followed by various processes leading to multi-systemic related symptoms. This abnormal metabolic state and the effects of a range of mediators such as products of oxidative and nitrosamine stress, may lead to progressive cell and metabolic dysfunction culminating in a hypometabolic state with low energy production. These processes do not seem to happen uniformly; although a spiralling of progressive inter-related and self-sustaining abnormalities may ensue, reversion to states of milder abnormalities is possible if the host is able to restate responses to improve homeostatic equilibrium.

Disease management and research efforts should seek to identify and apply strategies targeted at the different pathophysiological dysfunctions that characterise different disease stages. As disease presentation varies over time, no single case description, set of diagnostic criteria, or molecular feature is currently diagnostic for all patients at all times. While acknowledging its limitations due to the incomplete research evidence, we suggest the proposed framework may improve research design and health care interventions for people with ME/CFS.
\end{abstract}

Key words: Chronic Fatigue Syndrome, Chronic Illness, ME/CFS, Management, Research 


\section{Introduction}

The lack of progress in Myalgic Encephalomyelitis/Chronic Fatigue Syndrome (ME/CFS) research has been attributed to a range of factors, including the paucity of large, high quality, hypothesis-driven studies, and controversy around diagnosis. Without recognised and validated biomarkers or diagnostic tests, there is over-reliance on patient history for diagnosis, which is based on criteria with imperfect sensitivity and specificity (Nacul et al., 2017) and which ignore disease sub-groups. Furthermore, the lack of consistency in the choice and application of research case definition has led to problems with reliability and comparability of research findings (Institute of Medicine (IOM), 2015). An additional factor complicating diagnosis and standardisation of cases for research studies is the time-related variation in phenotype both in the short- (Ramsay, 1986; Twisk, 2018) and long-term (Reynolds et al., 2014), which has seldom been considered in research studies.

The heterogeneity of illness symptoms, severity, progression, and duration lends itself towards the categorisation of different phases or subtypes of ME/CFS to help identify predictors of prognosis. In some studies, female sex, age (Clark et al., 1995; Joyce et al., 1997; Kroenke et al., 1988), and socio-economic status (Bierl et al., 2004) have been attributed to poor prognosis, however the variable nature of both case or population sampling and diagnostic criteria has led to ambiguity of results and reinforced the need for ongoing research in this area (Jason et al., 2005). Further subtypes have been defined through genetic studies (Kerr et al., 2008; Zhang et al., 2010), metabolomics studies (Nagy-Szakal et al., 2018), on the basis of 'minor' symptoms (i.e. musculoskeletal, infectious, or neurological) (Janal et al., 2006), and, duration of disease studies (Reynolds et al., 2014), highlighting the multitude of possible ways ME/CFS patients can be categorised. Other studies have identified variations in symptom profiles as disease progresses however their results are often limited by cross-sectional study design (Friedberg et al., 2000), and/or recall bias (Chu et al., 2019). The breadth of subtype studies available follow a similar model of looking for patterns across patient groups at single time-points, far fewer are considering longitudinal subtyping and disease progression of a single patient cohort over time.

The concept of the natural history of disease is well understood in public health and medicine: many, if not all, diseases are framed using this construct to formulate how they progress from a pre-illness stage up to final disease outcome, which may vary from full recovery to death. A good understanding of the disease course is vital not only for the design of preventative and intervention studies (Jewell, 2016), but also to assess the timing and type of intervention that minimises disease risk or optimises prognosis. Although there is some understanding of the natural history of ME/CFS, this has been limited by problems in case definition (as above) as well as by the paucity of longitudinal studies, and in particular those that follow up individuals pre-illness. A review of studies on prognosis of CFS (Joyce et al., 1997) suggested recovery rates under $10 \%$ in adults, and an improvement rate over $40 \%$ for people with fatigue lasting less than 6 months. The prognosis was worse when: less stringent case definitions were used; in older people; in more chronic cases; and, in the presence of psychiatric co-morbidity. A subsequent systematic review on prognosis found a median recovery rate of 5\%, and median proportion of people improving of 39.5\% (Cairns and Hotopf, 2005) with many reporting symptoms still present at follow up.

This conceptual paper explores the long-term course of the disease and how presentation and pathophysiological abnormalities may vary with time. We consider the implications for case management and research design, including case recognition and treatment, and for the standardisation of studies' protocols and the interpretation of their findings. The pathophysiological concepts discussed are based on evidence from clinical observations and research, where available, and, as such, are not claimed to be 
original or indeed conclusive, but rather serve to highlight our proposed characterisation of ME/CFS's distinct stages within the concept of natural history of the disease. Table 1 summarises the proposed characterisation of disease stages in ME/CFS, which may be used in support of research design and the development of preventative, treatment, and rehabilitative interventions for this disease.

\begin{tabular}{|c|c|c|c|c|c|}
\hline Timing & No disease & Onset & $0-4$ months & 4 months* & 2 years $+\uparrow$ \\
\hline Stage & Predisposition & $\begin{array}{l}\text { Trigger and } \\
\text { pre-illness }\end{array}$ & $\begin{array}{l}\text { Prodromal } \\
\text { period }\end{array}$ & Early disease & $\begin{array}{l}\text { Established } \\
\text { disease }\end{array}$ \\
\hline $\begin{array}{l}\text { Clinical } \\
\text { phenotype }\end{array}$ & No symptoms & $\begin{array}{l}\text { Non-specific } \\
\text { or related } \\
\text { to triggering } \\
\text { "insult" }\end{array}$ & FCSt & $\begin{array}{l}\text { FCS } \$ \text { variable } \\
\text { severity and } \\
\text { progress }\end{array}$ & $\begin{array}{l}\text { Mild, } \\
\text { moderate, } \\
\text { severe and } \\
\text { complicated } \\
\text { disease }\end{array}$ \\
\hline Prevention level & $\begin{array}{l}\text { Primary } \\
\text { prevention }\end{array}$ & $\begin{array}{l}\text { Treatment of } \\
\text { "insult" \& } \\
\text { primary } \\
\text { prevention }\end{array}$ & $\begin{array}{l}\text { Secondary } \\
\text { prevention }\end{array}$ & $\begin{array}{l}\text { Treatment \& } \\
\text { secondary } \\
\text { prevention }\end{array}$ & $\begin{array}{l}\text { Treatment } \\
\text { and tertiary } \\
\text { prevention }\end{array}$ \\
\hline $\begin{array}{l}\text { Recovery } \\
\text { Potential § }\end{array}$ & & & Likely & Possible & Less likely \\
\hline Pathophysiology & $\begin{array}{l}\text { Predisposing } \\
\text { factors }\end{array}$ & $\begin{array}{l}\text { Non-specific } \\
\text { host response } \\
\text { and related to } \\
\text { specific } \\
\text { trigger factor }\end{array}$ & $\begin{array}{l}\text { Neuro- } \\
\text { immune } \\
\text { response to } \\
\text { insult and } \\
\text { fight for } \\
\text { homeostasis }\end{array}$ & $\begin{array}{l}\text { Neuro- } \\
\text { inflammation } \\
\text { and systemic } \\
\text { consequences; } \\
\text { aberrant } \\
\text { homeostasis }\end{array}$ & $\begin{array}{l}\text { Systemic } \\
\text { disease, } \\
\text { aberrant or } \\
\text { failed } \\
\text { homeostasis }\end{array}$ \\
\hline
\end{tabular}

* 3-6 months is commonly referred as the minimum period of symptoms before diagnosis is made in children and adults respectively (Shepherd and Chaudhuri, 2019)

$\dagger 2$ years is commonly referred (Nisenbaum et al., 2003; Russell et al., 2016), but this is variable, and depends on a range of factors, including individual response to early disease

$\ddagger$ FCS = fatigue-complex symptoms: initially predominantly neuro-immune and autonomic symptoms (prior to early disease), and variable systemic symptoms in established disease

$\S$ Tentative proportions for recovery are: likely $(>\mathbf{7 5 \%})$; possible $(<20 \%)$; less likely $(<5 \%)$. "Likely" and "possible" are based on recovery from arboviruses and EBV (Seet et al., 2007; White et al., 1998)); " less likely" is based on reviews on prognosis (Wilshire et al., 2017)

\section{Pathophysiological and cellular abnormalities following host exposure to "stressors" or "insults"}

Prior to exploring the course of ME/CFS, it is important to revisit some concepts related to mechanisms of disease that have been used in the context of other diseases and situations, and of homeostasis. In particular, we will focus on aspects of the pathophysiology of severe illness such as those in sepsis or poly-trauma. The benefits of considering the above examples are that acute injuries have been extensively 
studied, and the high intensity and speed of events result in changes that are easier to identify and characterise, such they may help guide this exploration of the mechanisms that might be taking place in $\mathrm{ME} / \mathrm{CFS}$.

The response to an insult involves multiple body-systems and has components that are independent of the aetiology of the insult and, to some extent, its severity. There are many commonalities between the response to sepsis and poly-trauma: both are acute and severe insults, to which many of the aspects of the host response are indistinguishable. Our proposal is based on the idea that there may be some similar mechanisms at play when individuals predisposed to ME/CFS are faced with a range of "insults" or "stressors". Needless to say, the hyper-acute changes and co-factors in both sepsis and poly-trauma occur in very rapid sequence, whereas in ME/CFS, physiological changes, even if they resemble those of acute injury in some respects, take place at a much slower pace with less obvious and less uniform patterns.

\section{Non-specific changes in response to severe acute injury}

This section refers specifically to the response mechanisms observed in sepsis and poly-trauma that we use as a template for attempting to understand what is likely to be occurring in ME/CFS. In both sepsis (Mello et al., 2016) and poly-trauma, (Keel and Trentz, 2005; Rosenblatt and Delmonico, 2016) a state of hyper-inflammation is observed initially as the host responds to the infection or traumatic stress with marked production of pro-inflammatory mediators, e.g. cytokines and polypeptides. A failing circulatory system is associated with activation of the hypothalamic-pituitary-adrenal (HPA) axis and increased sympathetic drive, contributing to metabolic changes and to increased energy expenditure (Herman et al., 2016; O’Donnell and Nacul, 2016).

In these conditions, the acute pro-inflammatory state is usually followed by a compensatory antiinflammatory response, with a different profile of biochemical and molecular mediators. The success of the host response in balancing pro- with anti- inflammatory responses, alongside injury-related factors, are key to long-term outcomes. The direct and indirect effects of immune response (some of which cause pathology if present in excess) contribute to a number of physiological changes, including those leading to the formation of reactive oxygen species (ROS, oxidative stress) and reactive nitrogen species (RNS, nitrosative stress). Endothelial and parenchymal cell damage may result because of a combination of factors, and may be secondary to polymorphonuclear leukocyte infiltration or to the action of reactive oxygen and nitrogen species, cytokines, vasoactive amines and other products.

Endothelial dysfunction results in capillary leakage, accelerated inflammation, platelet aggregation, coagulation, and loss of vascular tone (Martin et al., 2016); vascular dysfunction is associated peripheral vasodilation due to increased nitric oxide and prostacyclin synthesis (Russell et al., 2018), a decrease in the proportion of perfused vessels and an increase in the heterogeneity of blood flow distribution (Pool et al., 2018). This results in relative hypovolemia, decreased capillary flow, haemo-concentration and microthrombi formation, and further contributes to reduced exchanges of oxygen and nutrients at the microcirculatory level. The consequent decreased cellular oxygen delivery eventually leads to cytopathic hypoxia. Adenosine triphosphate (ATP) increased consumption and ensuing deficits cascade into a range of metabolic disturbances with systemic effects (Singer, 2014), and promote changes in membrane permeability that lead to dysfunctional transmembrane ion transport. In acutely and severely ill patients, reperfusion results in further oxidative damage (Kalogeris et al., 2012; O'Donnell and Nacul, 2016). Additional failures of biological and cell processes lead to multiple dysfunctions and systems and organ failure, and potentially irreversible disease (O’Donnell and Nacul, 2016). 


\section{Evidence of abnormalities in ME/CFS and loss of normal homeostasis}

Concepts that are relevant here are those of homeostasis and allostasis. While homeostasis refers to the "stability of physiological systems", allostasis has been defined as "the adaptive processes aimed to maintain homeostasis following acute stress, and which contribute to wear and tear on the body and the brain, or allostatic overload" (McEwen, 2005). A central characteristic of individuals with ME/CFS points to a state of homeostatic failure (Hatziagelaki et al., 2018), aggravated by the incidence of or increase in levels of new stressors or by the increase in allostatic load (Arroll, 2013). Typical stressors include infection (see references reviewed by Shepherd \& Chaudhuri, pages 17-21) (Shepherd and Chaudhuri, 2019), physical and mental exertion, and a range of environmental and individual factors (Arroll et al., 2014; Arseneau et al., 2017; Brown et al., 2015; Centers for Disease Control and Prevention, 2006; Hu and Baines, 2018; Keller et al., 2014).

In those who do not develop ME/CFS or prolonged illness following an acute infection, external stressors may initially cause physiological changes accompanied by non-specific symptoms, but the state of homeostatic equilibrium that operated before the insult is quickly restored. Failing re-establishment of this equilibrium, there may be a shift to a state of 'aberrant homeostasis', where physiological processes converge to a new or alternative state of functioning; a state that remains homeostatic in nature, but functions at a less optimum level (Klimas et al., 2012). While such a state may be adequate for many physiological processes, it will be inadequate or inefficient for a number of other processes and functions and the prolongation of such aberrant functioning will itself represent another potential source of ongoing stress.

There is a growing body of evidence on biological abnormalities in ME/CFS that have been reviewed elsewhere (Edwards et al., 2016; Monro and Puri, 2018; Shepherd and Chaudhuri, 2019), and summarised by Komaroff (2019). Of note, many of the abnormalities shown in severe injury have also been identified in ME/CFS such as: immune dysfunction, including pro-inflammatory response (especially at early stages of disease) (Cliff et al., 2019; Lord et al., 2014); autonomic nervous system (Cambras et al., 2018; Esterov and Greenwald, 2017; Oosterwijck et al., 2017); HPA axis dysfunction (Tomas et al., 2013); hypovolemia (van Campen et al., 2018); nitrosamine and oxidative stress (Newton et al., 2012); endothelial dysfunction (Newton et al., 2012); metabolic dysfunction (Germain et al., 2018); dysfunction of membrane transport (Chaudhuri et al., 2000); and, tissue hypoxia (Vermeulen and Vermeulen van Eck, 2014).

\section{The stages of ME/CFS}

Another tool widely used in clinical medicine is staging systems. Using sepsis again as an example, such a system was proposed at the International Sepsis Definitions Conference in 2001 to introduce the stratification of patients with sepsis (Levy et al., 2003). By applying PIRO (predisposition, infection/insult, response, and organ dysfunction) patients were carefully stratified into the appropriate subgroups allowing for more accurate prognostication in emergency medical services (Rathour et al., 2015). The idea of classifying people with ME or CFS into distinct categories or stages has been explored previously by several theorists. One school of thought proposes categories based on the psychological process of coming to terms with this new and evolving state of health rather than addressing biological differences, and are defined as such by the emotions common to any trauma experience: e.g. denial, fear, frustration, and acceptance (Fennell, 1995; Jason et al., 1999). Alternatively, Schweitzer (1999) proposes the different forms of CFS according to more physical categories (Prodrome, Relapse and Remission, 
Improvement and Plateau, and Collapse followed by slow worsening with no remission) that we aim to expand on, as follows.

We show a tentative representation of the key pathophysiological mechanisms operating in each stage of ME/CFS in Figure 1. As it happens after severe injury or sepsis, the range and order of occurrence of biological processes taking place in ME/CFS may vary, as may their relative significance and impact on each individual. Therefore, it is important to note that although the various abnormalities may be occurring continuously and often simultaneously, the predominance of specific dysfunctions varies over time and from individual to individual (see Table 1 and Figure 1).

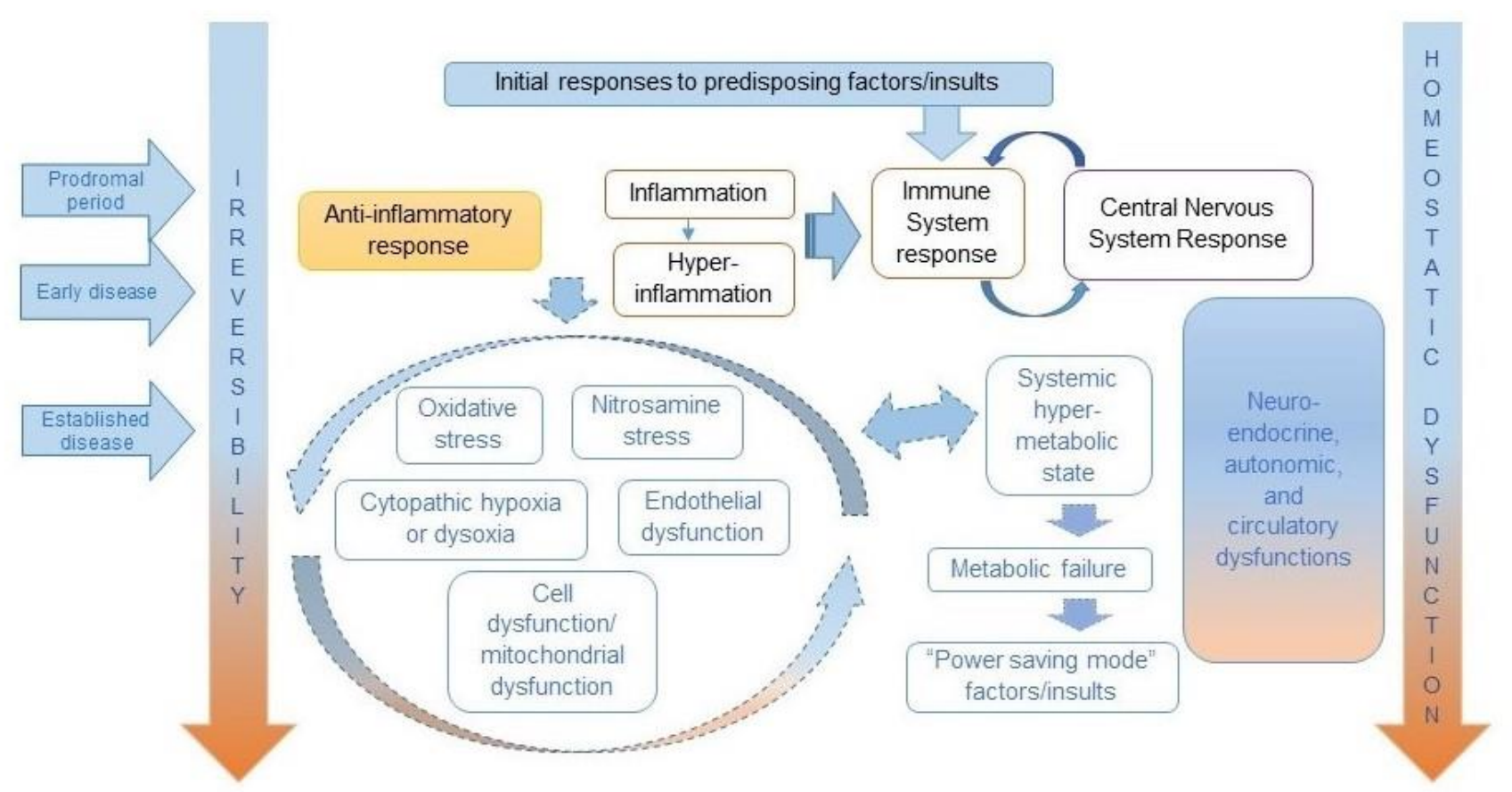

Figure 1. Hypothesised key pathophysiological mechanisms for ME/CFS

\section{Predisposition and triggering of disease}

Individuals with a combination of genetic predispositions and exposures to environmental factors may first manifest symptoms of ME/CFS following their encounter with a specific trigger, of which acute infections of various aetiologies are the most commonly reported; other patients report a more insidious onset with no obvious initiating factor (Shepherd and Chaudhuri, 2019). While it remains unclear exactly which individuals are predisposed to develop ME/CFS, and why, some patterns have emerged. For example, gender- and age-specific factors are thought to contribute to the risk of ME/CFS (Bakken et al., 2014), with epidemiological studies consistently reporting higher rates of the disease in females (Nacul et al., 2011; Prins et al., 2006). Although most cases are endemic, there have been reports of epidemic cases, which suggest infectious or another environmental cases have a role (Levine et al., 1992, 1997; Monro and Puri, 2018; Naess et al., 2012). Cases are predominantly reported in North America, Europe and Oceania; however, the occurrence of ME/CFS is thought to be global with evidence of cases in other parts of the world (Cho et al., 2009; Nacul et al., 1999; Njoku et al., 2007). The role of genetic variation has been supported by a number of family-based studies assessing the possibility of a heritable component 
(Albright et al., 2011; Buchwald et al., 2001; Walsh et al., 2001). Genes underpinning immune system function and inflammatory response may contribute to genetic susceptibility for ME/CFS; some studies suggest associations with human leucocyte antigen class II alleles (Keller et al., 1994; Smith et al., 2005) and in genes related to the complement cascade, chemokines, cytokine signalling, and toll-like receptor signalling (Rajeevan et al., 2015). Small genome-wide association studies (GWAS) have had little overlap in results save for two SNPs in the GRIK2 gene: a gene implicated in a number of neurological conditions such as autism and schizophrenia (Smith et al., 2011); in the GRIK3 gene: relating to a pattern recognition receptor capable of binding to a broad range of pathogens; and in the non-coding regions of T-cell receptor loci (Schlauch et al., 2016). A further study reported SNP markers in candidate genes involved in hypothalamic-pituitary-adrenal (HPA) axis function and neurotransmitter systems distinguished individuals with ME/CFS (Smith et al., 2006).

\section{Prodromal period}

In addition to any manifestations specifically related to the acute insult or triggering event, the mechanisms involved in producing the first symptoms of ME/CFS may be similar to what has been described in relation to "sickness behaviour" (Low et al., 2015), or, in those with severe acute disease, "systemic inflammatory response syndrome" (Mello et al., 2016). These result from the interaction of an infective agent or other insult with the immune system, and their effect on the central nervous system.

The immune system-nervous system interactions involve bidirectional signals (Black, 1994; Prüss et al., 2017; Wrona, 2006): immune system activity may interfere with central nervous system (CNS) function via various mechanisms, e.g. release and action of pro-inflammatory cytokines and other mediators, while various neurotransmitters, neuropeptides and neuro-hormones may affect immune function. The HPA system and the autonomic nervous system (ANS) are affected, with consequences that may be observed well beyond the CNS.

These effects may vary according to different factors, such as host susceptibility, the nature and persistence (or return to normality) of systemic and local immune dysfunction, altered CNS metabolism, neuro-transmission, brain perfusion changes, and the integrity of the blood-brain barrier (Bested et al., 2001; Chaudhuri and Behan, 2000; Natelson et al., 2005; Schutzer et al., 2011).

Particular characteristics of the specific infectious agent or stressor may play a role, which would explain the different risks of disease development following acute infection. For example, there has long been an interest in the association between ME/CFS and infections such as Epstein-Barr virus (EBV) and other herpesviruses. Herpesviruses tend to be neurotropic and persist following acute infection in a latent state. Similar to EBV infection (White et al., 1998), the risk of chronic fatigue has been shown to be substantially increased following viral meningitis, a relatively severe infection of the central nervous system (CNS) (Hotopf et al., 1996). The current diagnostic methodology of ME/CFS stipulating the presence of symptoms for more than six months (Carruthers et al., 2003; Fukuda et al., 1994), and the absence of a positive validated diagnostic test makes the above processes (occurring pre-diagnosis) difficult to substantiate from existing biomedical research.

\section{Early Disease}

Early disease represents a continuation of the processes initiated at the prodromal period, when there is a failure of physiological and homeostatic processes to resume previous levels of equilibrium and normality. Fatigue and other symptoms may be largely explained by a combination of the local and systemic effects of pro-inflammatory and other mediators or toxins, CNS metabolic dysfunction (with enhanced excitability and other changes), and a systemic hyper-metabolic state. With higher energy demands for essential biological processes, there will be a reduction in the available energy for less essential tasks, 
such as those demanding increased physical or mental exertion. Fatigue, post-exertional malaise (PEM) and sleep problems are quite marked, particularly in the most severe cases. Autonomic, neuro-endocrine and circulatory dysfunctions are present and may cause significant symptoms, though they may gain more prominent pathophysiological roles as disease progresses. The increased production and action of antiinflammatory mediators, and their ability to counter-balance pro-inflammatory stimuli, modulate physiological responses and symptoms and affect disease progress or reversibility. As mentioned previously, without a validated biomarker to diagnose ME/CFS early it is again difficult to substantiate the exact mechanisms occurring in the early disease phase. Research into potential diagnostic markers, such as the recent study on impedance signatures (Esfandyarpour et al., 2019), are crucial not only clinically, but to identify these mechanisms as possible targets for early intervention.

\section{Established ME/CFS}

The persistence of immune and CNS dysfunction with the initial over-production of pro-inflammatory and neurotoxic factors may result in a prolonged state of low-grade neuro- and systemic inflammation. In the CNS, a status of glial activation with microglial hypersensitivity to peripheral (Rowe et al., 2016) and regional stimuli is established (Black, 1994; Glassford, 2017; Rowe et al., 2013, 2016), akin to what has been described in chronic pain states (Dubin and Patapoutian, 2010). In support of central nervous system dysfunction, neuroimaging studies have shown various abnormalities in ME/CFS, often associated with symptoms of fatigue and other indications of severity (Morris et al., 2018). Glial activation in several areas of the brain has also been demonstrated in PET scans of patients with fibromyalgia, compared to controls, which was correlated to the severity of fatigue (Albrecht et al., 2019; Morris et al., 2018) Neuro-glial bidirectional signalling is associated with increased production of neuro-excitatory neurotransmitters and immune-inflammatory mediators (Glassford, 2017). Nervous system dysfunction affecting parts of the brain, brain stem, and autonomic nervous system, could explain not only the encephalopathic or neurocognitive type of symptoms, but also those resulting from disruption of key central regulatory mechanisms, such as those involved in endocrine, circulatory, thermoregulation, and respiratory control (Beaumont et al., 2012; Cambras et al., 2018; Chu et al., 2019; Glassford, 2017; Shepherd and Chaudhuri, 2019). Examples of these include intolerance to extremes of temperature, chills and temperature variations, intolerance to exertion, hyperventilation or irregular breathing, orthostatic intolerance, hypotension, postural orthostatic tachycardia and other symptoms related to autonomic and endocrine control function (Carruthers et al., 2003).

Among the various by-products produced as a consequence of ongoing abnormalities, are highly reactive ROS and NOS or free radicals, which affect cell signalling and cell functioning and structure, particularly when present at high levels. It has been hypothesized that free radicals, and increased levels of nitric oxide and peroxynitrite in particular, play a significant role in ME/CFS (Pall, 2000, 2003); their links to immune and neuro signalling, cell integrity, mitochondrial function and energy metabolism may play an important part in the long term abnormalities in ME/CFS.

The nature of neuro-immune and other dysfunctions may change as disease progresses. While a proinflammatory state is typical of the early response to insults, immune abnormalities may become less marked (and less pro-inflammatory) with time (Hornig et al., 2015), and patients with longer periods of illness may show fewer inflammatory immunological abnormalities. Similarly, autonomic and endocrine abnormalities, some of which may be very marked in the initial stages, may be replaced with more chronic neuro-endocrine and circulatory manifestations. In persistent non-severe cases, neuro-cognitive, autonomic and musculoskeletal symptoms tend to become more severe with time; in more favourable cases, these may instead decrease as a consequence of the success of the host's compensatory mechanisms. 


\section{Long-term, advanced and complicated disease}

As the disease progresses, physiological and systems abnormalities take their toll and cell dysfunction becomes more pronounced. Endothelial dysfunction may arise as a consequence of a range of factors, including, but not limited to, persistent oxidative and nitrosative stress and circulatory dysfunction (Bell, 2007; Maes et al., 2011; Monro and Puri, 2018; Newton et al., 2012; Pall, 2000). The associated reduced delivery of oxygen and nutrients to the cell, leads to deterioration of cell function and impaired energy metabolism (Bell, 2007; Germain et al., 2017; Rutherford et al., 2016) and a decreased ability of the cell to extract oxygen and produce energy, a condition known as cytopathic hypoxia. As suggested by Naviaux et al. (2016), in cases of ME/CFS with mean duration of symptoms over 17 years, there is a shutting down of various metabolic processes leading to a hypometabolic state, i.e. a move to an energy-saving mode. At this stage, symptoms are likely to be severe, with profound fatigue, intolerance to effort, PEM and other systemic symptoms, which are largely explained by the slowness of physiological and metabolic processes and decreased energy production.

\section{Disease severity and reversibility}

It is unknown how the initial host response to a stressor or insult compares in individuals who do or do not develop typical symptoms of ME/CFS. However, the return to good health, which happens to most people following exposure to mild or moderate levels of insult, seems to be impeded in ME/CFS as symptoms persist for longer than six months and this time interval became featured in some of the currently used diagnostic criteria (Carruthers et al., 2003; Fukuda et al., 1994). This suggests that subsequent mechanisms involved in the host response will differ at some point in those who develop ME/CFS from those who regain full health. Therefore, a key question is what determines full recovery or, alternatively, the perpetuation and transformation of symptoms.

While the abnormalities observed in acute disease are general and mostly reversible once the challenge from the stressor ceases, some degree of dysfunction may persist for longer periods. The degree of reversibility of various physiological abnormalities is likely to decrease with time, and some permanent functional, and even structural, damage may occur consequently. This is likely caused by any or a combination of the persistence of the initial stressor, an accumulation of insults, or a continuing dysfunctional host-response.

The pathophysiological distinction between cases from the milder to the more severe end of the ME/CFS spectrum may relate to near-normal homeostatic regulation in milder cases, and established homeostatic dysregulation or 'aberrant homeostasis' with multi-systemic consequences in moderate to severe cases. Alternatively, homeostatic failure with variable multi-system physiological failure and increasing degrees of irreversibility may happen in the most severe cases.

The early stage of ME/CFS is of variable duration, but is usually considered to be between 4- 6 months to 2 years after the start of prodromal symptoms. Reversibility is possible, but often people will evolve to chronicity, or established ME/CFS, with either: a) partial reversal of dysfunctional physiological mechanisms (mild cases with slow improvement over time); b) persistence of dysfunctions and symptoms (mild or moderate cases with stable symptoms or slow changes over time); or c) worsening dysfunctions and symptoms (moderate and severe cases) (CFS/ME Working Group, 2002). Note that some cases 
present early with severe symptoms, which not uncommonly evolve to a milder form (National Institute of Clinical Excellence, 2007).

One way of thinking about these phases is as interconnected spirals, each representing a distinct disease phase. Individuals may either remain for long periods in a single phase with symptoms fluctuating within the "spiral section" or move between phases either upwards (i.e. towards better health status) or downwards (i.e. towards disease deterioration). Figure 2 represents an illustration of the multi-spiralling disease course suggested for ME/CFS, and shows how patients may move across spirals, with different molecular and system abnormalities.

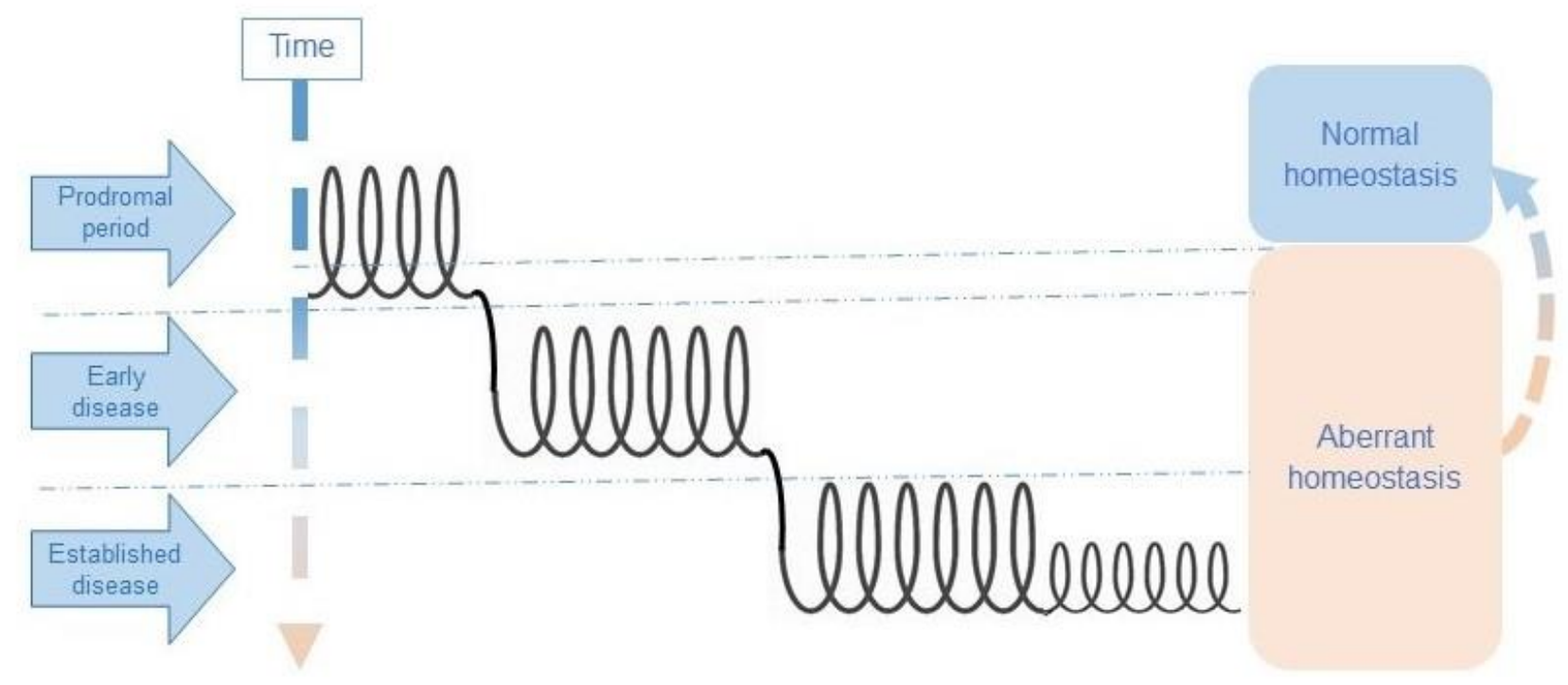

Figure 2. Hypothetical stages of disease in ME/CFS

\section{Management approaches}

Although not representing perfectly defined discrete phases, conceptualisation of disease stages helps to understand disease pathways, their operation and interconnections along the disease course, and therefore they may support the planning of research and health interventions.

Until there is conclusive evidence on specific pathophysiological mechanisms and effective treatments, disease management should rely on a strong health professional-patient relationship with regular followups and a cautious degree of trial and error in treatment approaches; such management should be informed by symptom characteristics and progression according to the stage of the disease. These should be based on ongoing dialogue and partnership between professionals and patient, extending to carers and family, with the involvement of the educational, occupational and social sectors as appropriate.

\section{Primary prevention}

While risk factors specific to ME/CFS are still largely unclear, it is very difficult to conceptualise or put in practice evidence based primary prevention strategies. As understanding of risk factors evolve, there is no reason why the approach to the understanding and the practice of ME/CFS medicine should be 
dissimilar to that of any other chronic disease. Nevertheless, it is reasonable to assume that in the face of acute infections or other insults, individuals should avoid exposure to further stresses, and periods of relative rest and pacing of activities may be appropriate to facilitate recovery from acute illness. This may require support from employers and teachers, for example, as appropriate (see Section below for the role of "presenteeism"), but prevention of common infections is not thought to be relevant in the context of ME/CFS prevention. For cases that might be triggered by environmental contaminants, such as chemicals, environmental protection policies and regulation may be important (Nacul et al., 2009).

Any variation in practice will come from a greater understanding of risk factors specific to ME/CFS and from the design of a disease-specific strategy for ME/CFS prevention, which is currently lacking. This strategy, if feasible, should be thought within the context of wider determinants of health (Dahlgren and Whitehead, 1991), using a model that applies to the prevention of chronic diseases in general.

\section{Secondary prevention at the prodromal and early disease phases}

Secondary prevention refers to early detection of a disease and early intervention, with the aim of reducing morbidity (Last, 2014). In the case of ME/CFS, early diagnosis could have an impact on disease management, even in the absence of specific treatment. In order to facilitate this, a provisional diagnosis of ME/CFS could be considered earlier, e.g. with two or three months of symptoms, with efforts made to encourage follow up appointments, until confirmation at four to six months, followed by further regular reviews (Shepherd and Chaudhuri, 2019).

Approaches towards earlier diagnosis would require a major shift from the healthcare professionals' perspectives, but it would serve to appropriately manage early symptoms and prevent disease deterioration. For example, at this stage when a diagnosis of ME/CFS is apparent, we would recommend a reduction of allostatic load, including sensible reduction in activity levels (Natelson, 2008; National Institute of Clinical Excellence, 2007; Rowe et al., 2017), avoidance of further stressors, and treatment of the infection or trigger factor(s) where possible. In addition, any specific therapies should be aimed at corrections of immune, CNS, and other dysfunctions, alongside prevention of complications.

Strong support at educational institutions and workplaces should be widely available, including consideration of the benefits for organisations and individuals of enabling proper recovery time away from, or with reduced time at, work or studies during illness (Higginbottom, 2018). Higginbottom refers to the growing evidence pointing out the higher costs of presenteeism (i.e. being physically at work, even if ill) compared to absenteeism, looking at the adverse effects on an individual's own health and productivity when turning up to work ill (Stewart et al., 2003). Employing this strategy might require society as a whole to recognise the importance of the needs of the individuals during illness for adequate recovery periods and of taking the pressure off individuals to be productive or present in their workplaces or classroom during these times. This would avoid or minimise negative impacts on their health in the short- and long- term, as increased or persistent exertion, whether it is physical or mental, may result in the worsening of symptoms and a delay in recovery (Shepherd and Chaudhuri, 2019).

\section{Established ME/CFS (Treatment, Tertiary prevention and Rehabilitation)}

Management of established ME/CFS should centre on the restoration of a healthier homeostatic balance through specific treatments and avoidance of aggravating factors. Due to the current insufficient evidence on specific treatments aimed at the pathophysiological abnormalities, management has been limited to life-style changes, such as "pacing", including advice on planning and executing activities within the individual's energy limit levels (Jason et al., 2008) and avoidance of other causes of neuro-immune 
overload; and symptomatic medications, such as analgesics and sleep medications (Carruthers et al., 2003). As evidence grows, treatments targeting multi-systemic abnormalities will have a place, such as those resulting from dysfunctions in the immune, neuro-endocrine, autonomic, circulatory and neuromuscular systems. Examples could include immune-based treatments, and those targeting oxidative stress and metabolic abnormalities. Tertiary prevention and rehabilitation are important here. Tertiary prevention refers to actions aiming at reducing the impact of long term illness and resulting disability (Last, 2014), including through rehabilitative interventions. The absence of an evidence based curative treatment should not deviate the main aim of supporting the individual and management of symptoms and disability.

A relatively new concept, that of quaternary prevention proposed originally by Jamoulle (2015), has been reviewed by Martins et al (2018) conceptualizing it as "the action taken to protect individuals (persons/patients) from medical interventions that are likely to cause more harm than good": the aim being "to reduce over-medicalisation and iatrogenic harm". With the wide range of, often non-evidence based, treatments used for ME/CFS including in alternative health practice (Alraek et al., 2011), and with the controversies in behavioural based therapies for ME/CFS (Marks, 2017), it is important that health professionals share the responsibility with patients in guiding them with personalised treatments. Decision-making should be well informed in relation to availability or lack of evidence, potential benefits and risks of treatments, with additional considerations about individual and service costs of such treatments.

\section{Severe or complicated ME/CFS}

Treatment for severe or complicated ME/CFS is more challenging, as patients may have achieved an advanced state of homeostatic dysregulation with increasing multi-system dysfunction and multi-systems complications. The previous state of chronic inflammation may now be subdued, while the body may enter a hypometabolic state (Naviaux et al., 2016). This includes the slowing of physiological pathways and reduction of energy output, with chronic cell and system malfunction. In addition to the measures used in early and less severe cases, any treatment approach needs to consider specific mechanisms leading to and perpetuating cell dysfunction (e.g. those associated with endothelial dysfunction and cytopathic hypoxia). This may include strategies aimed at reverting neuro-endocrine and metabolic abnormalities and at rehabilitation. Examples of interventions include neurological rehabilitation (e.g. gentle physiotherapy), nutritional rehabilitation (which might need to involve enteral feeding), and those targeting circulatory dysfunction (e.g. treatment of hypotension and postural tachycardia and other manifestations of orthostatic intolerance, and the various consequences of prolonged illness (e.g. screening for and treatment of osteoporosis). Treatment approaches targeting specific energy metabolic dysfunctions, as well as specific nutritional and hormonal supplements, may also play a restorative role. However, these still need development and validation before they can be used beyond individually tailored approaches. Severely affected patients have their activities considerably restricted, often struggling with self-care, and needing support from carers and from a multi-disciplinary health-team. For this sub-group of patients, effective input and support from social, educational and occupational health services may be even more important, alongside a range of rehabilitative interventions.

\section{General and tailored approaches}

The nature of persistent dysfunctions, and whether or not they can be controlled or resolved, may be central to prognosis and treatment in ME/CFS. For example, in those with more severe post-exertional symptoms (more likely affected by autonomic, neuro-endocrine, and energy metabolism dysfunction), 
energy management, through pacing and sensible rest, may be essential to allow the body to enact its recovery potential, together with specific treatments to address systems dysfunctions. Those with long illness duration, but with milder symptoms which are improving, may become less fatigued and more tolerant to exertion, feel more energetic and have less cognitive dysfunction or "brain fog"; their postexertional symptoms may be less pronounced or limited to major activities. Such cases could benefit from a program of individually tailored, paced, stepwise and increasing exposure to activities. However, those in whom disease is progressing unfavourably may benefit from medical treatments targeted at specific dysfunctions alongside rehabilitation. As in many chronic debilitating diseases, psychological therapies may have a role in supporting individuals through their chronic illness as part of an important supplementary component of a holistic medical care that includes a personal approach to management and treatment.

\section{Research at different disease phases}

A number of research questions still need answers, requiring different strategies and ways of selecting research participants. Researchers should consider the advantages of restricting the study population of cases to those who meet diagnostic criteria with higher specificity (Nacul et al, 2017) and of case stratification, including sub-grouping of cases into disease stages, or alternatively focusing on a specific stage.

While there is no doubt that molecular research is essential to revealing disease pathways and for biomarker discovery, other types of research, such as clinical, epidemiological, environmental, health services, policy and education are essential for better disease recognition, prevention, diagnosis and treatment. Furthermore, we argue that researchers should consider the reproducibility of their studies, particularly when applying new technology, to avoid potential pitfalls such as those considered by VanElzakker et al. (2019).

Within the field of ME/CFS research, studies using small participant numbers and variable diagnostic criteria are commonplace, and lead to non-replicable or non-comparable results. The ongoing study of risk factors may further benefit from existing large datasets (e.g. GP electronic health record databases for research (https://www.pcrd.purdue.edu/ )); or of general (e.g. UK Biobank (https://www.ukbiobank.ac.uk/ )), or disease specific databases and biobanks (e.g. UKMEB: https://cureme.lshtm.ac.uk/; Solve ME/CFS Initiative: https://solvecfs.org/). Bioresources such as the UKMEB, that use a strict protocol for the recruitment of participants, collection and storage of biosamples (Lacerda et al., 2017) would serve to improve the replicability of studies by minimizing the variation in sampling and use of diagnostic criteria, and further serve for validation studies. There is also a role for existing cohorts with access to patients for diagnostic confirmation, and the application of life course epidemiology methods (De Stavola et al., 2006) using retrospective or prospective longitudinal designs. Approaches may target individual, environmental or genetic factors. Further genomic association studies could help the investigation of the association of candidate genes with disease, based on hypotheses inspired by the evolving understanding of disease mechanisms at the molecular level; genetic family studies and large genome wide association studies could also contribute to the body of knowledge.

Information on risk factors for ME/CFS are currently scarce, though these would be very useful for primary prevention. Thus, we recommend considering the research approaches used in the study of other chronic diseases, as we may gain new insights on familial and individual risks, including genetic, 
environmental, and life-style factors. Examples of proxy models that could be used to further our understanding of risk factors and the immune response in ME/CFS include interferon-alpha (IFN-a) treatment for hepatitis C (Russell et al., 2019), and cohort studies that follow fatigue after infection with Epstein-Barr Virus (EBV) (Katz et al., 2009). Considering IFN-a as the 'trigger' in the first case allows for observation and tracking of the disease profile prior to, during, and after the presence of the insult while following cohorts of patients from an early stage further allows the identification of possible risk factors. While appreciating that distinct mechanisms may be at play in ME/CFS, it is reasonable to consider similar proxy models to seek further understanding of immune profiles and response to insults in fatiguing diseases.

Looking at other models of disease that include 'invisible stages' of illness brings us to the change in focus that evolved in the study of Alzheimer's disease. Over the past decade, a conceptual shift to consider the disease as a continuum has occurred and, along with the discovery of biomarkers, has re-focused the research agenda towards the pre-clinical stage and early intervention (Dubois et al., 2016). Similarly, we suggest that a re-focus of the ME/CFS research agenda towards the pre-clinical stage by way of larger cohort studies, may contribute to revealing potential risk factors to support primary prevention efforts.

In relation to secondary prevention, research could focus on the pathophysiology of early disease and early interventions at sub-clinical, acute, and early disease stages; and, target those factors that facilitate and hamper recovery from acute disease. This may require follow up of relatively large cohorts of individuals from the moment they are exposed to an insult - such as an acute infection (Katz et al., 2009), to distinguish those who develop prolonged, chronic fatigue and ME/CFS. Studies could also recruit and follow individuals presenting with fatigue for shorter periods prior to being symptomatic for a long enough period for a diagnosis of ME/CFS be considered (i.e. usually 6 months).

For established disease, research efforts should target the understanding of mechanisms leading to the perpetuation of abnormalities and pathways to recovery, including specific treatments targeted at various system and molecular abnormalities. Again, longitudinal studies are essential to address temporal pathophysiological changes to guide therapeutic approaches at different disease stages, and to investigate short- and long-term complications, including co-morbidities and mortality. Younger patients with a shorter duration of disease have been found to present with different phenotypes, such as in relation to autonomic nervous system manifestations (Reynolds et al., 2014) and therefore are more likely to require specific treatment for postural orthostatic tachycardia syndrome (PoTS) or postural hypotension symptoms. Description and/or comparisons of specific subtypes through longitudinal studies would help determine differences in phenotype and encourage a more tailored approach to treatment management. Research on perceptions and attitudes to prolonged illnesses, from the individual, family, educational, occupational, and wider societal points of view also has a conducive role to secondary prevention.

Tertiary prevention should focus on rehabilitation strategies based on the understanding of the pathophysiology of severe and complicated disease and disability, and interventions at individual, service, and societal levels.

An important consideration in the search for evidence in ME/CFS was signalled by Bell over 20 years ago (2007), who wrote: "we need to change the focus of our telescope from looking at large organs to looking at single cells". As we focus on the cellular level of molecular and systems medicine, and transfer knowledge acquired from other conditions (such as acute severe injury), we should get closer to finding 
the real explanations for the various subgroups in ME/CFS. The "omics" technologies - e.g. transcriptomics, metabolomics, proteomics and genomics, including pharmacogenomics - are becoming increasingly accessible. Meaningful and translatable research outputs are now possible based on relevant research questions, as long as strong methodological approaches are applied at all research phases. These should cover research design and case selection, sampling and management of bio-specimens, and appropriate application of technology and interpretation of findings.

As disease understanding evolves, we should move closer to personalized health care and medicine and more specific strategies for prevention and treatment will become possible (Haendel et al., 2018). Examples of such strategies may include the targeting of high-risk individuals for screening, diagnosis and treatment; molecular diagnoses of subgroups, and targeted treatment according to molecular subtypes; keeping in mind the importance of quaternary prevention, to balance the need of finding the best evidence with that of promoting well-being of patients.

\section{Conclusions}

Discrepancies in the use of diagnostic criteria and sampling methods have led to much variation in research results in ME/CFS and this is mirrored in the care of those affected. As research is directed towards biomedical, systems and molecular investigations, the need for better disease stratification becomes more evident, for both research purposes and clinical practice. It is important, therefore, to consider ME/CFS as a continuum and the different stages patients go through during the course of their disease, their severity, and the presence and degree of complications as key parameters for stratification.

Pathophysiological patterns and changes along and across disease stages result in the expression of different, albeit overlapping phenotypes. Therefore, the approach to diagnosis, subgrouping, and clinical management will vary according to these phenotypes, as will research questions and the selection of patients for research. Loss of specificity caused by the amalgamation of people with ME/CFS with those with chronic fatigue due to other causes in observational and interventional studies is problematic (Nacul et al., 2017; White et al., 2011). Similarly, ignoring different subgroups of ME/CFS - including those related to disease stage, may have an impact on the outputs and the interpretation of research investigating disease mechanisms, pathways, and clinical trials. Moreover, researchers should strive to design reproducible studies, particularly when using new technologies.

Both the parallels with other chronic diseases and the concepts of determinants of health and levels of intervention are useful, as they provide a framework that can be used to guide disease prevention and management, as well as research direction. The recruitment of individuals for research at pre-illness stage could be invaluable to understanding the biological mechanisms at play before, during and after an insult. Longitudinal studies would help determine where individuals are in terms of the natural course of the disease and encourage the investigation abnormalities and treatments that take into account disease stage, here considered as an additional category for subtyping.

This paper sought to provide a simple framework, in line with other diseases, to help re-focus efforts of research and treatment management. Regardless of our current understanding of ME/CFS, common sense, acquired transferrable knowledge, and good health care are required to ensure safe, high quality care for those who are ill. 


\section{Declaration of interests}

All authors declare they have no conflicts of interests.

\section{Funding statement}

Research reported in this manuscript was supported by the National Institutes of Health under award number 2R01AI103629. The content is solely the responsibility of the authors and does not necessarily represent the official views of the NIH. 


\section{References}

Albrecht DS, Forsberg A, Sandström A, et al. (2019) Brain glial activation in fibromyalgia - A multisite positron emission tomography investigation. Brain, Behavior, and Immunity 75. Academic Press: 72-83. DOI: 10.1016/J.BBI.2018.09.018.

Albright F, Light K, Light A, et al. (2011) Evidence for a heritable predisposition to Chronic Fatigue Syndrome. BMC Neurol 11. 2011/05/31.: 62. DOI: 10.1186/1471-2377-11-62.

Alraek T, Lee MS, Choi T-Y, et al. (2011) Complementary and alternative medicine for patients with chronic fatigue syndrome: a systematic review. BMC complementary and alternative medicine 11. BioMed Central: 87. DOI: 10.1186/1472-6882-11-87.

Arroll MA (2013) Allostatic overload in myalgic encephalomyelitis/chronic fatigue syndrome (ME/CFS). Medical Hypotheses 81(3): 506-508. DOI: 10.1016/j.mehy.2013.06.023.

Arroll MA, Attree EA, O'Leary JM, et al. (2014) The delayed fatigue effect in myalgic encephalomyelitis/chronic fatigue syndrome (ME/CFS). Fatigue: Biomedicine, Health \& Behavior 64. Routledge: 393-394. DOI: 10.1080/21641846.2014.892755.

Arseneau L, Ko G, Elgez A, et al. (2017) Environmental Exposures as a Potential Underlying Factor in Chronic Fatigue Syndrome; a Case Report. Medical Research Archives 5(12). DOI: 10.18103/mra.v5i12.1573.

Bakken IJ, Tveito K, Gunnes N, et al. (2014) Two age peaks in the incidence of chronic fatigue syndrome/myalgic encephalomyelitis: a population-based registry study from Norway 2008-2012. BMC Med 12(1): 167. DOI: 10.1186/s12916-014-0167-5.

Beaumont A, Burton AR, Lemon J, et al. (2012) Reduced Cardiac Vagal Modulation Impacts on Cognitive Performance in Chronic Fatigue Syndrome. PLoS One. 7(11): e49518. DOI: 10.1371/journal.pone.0049518.

Bell DS (2007) Cellular Hypoxia and Neuro-Immune Fatigue. 1st ed. Livermore: WingSpan Press.

Bested AC, Saunders PR and Logan AC (2001) Chronic fatigue syndrome: neurological findings may be related to blood--brain barrier permeability. Medical hypotheses 57(2): 231-7. DOI: 10.1054/mehy.2001.1306.

Black PH (1994) Immune system-central nervous system interactions: effect and immunomodulatory consequences of immune system mediators on the brain. Antimicrobial Agents and Chemotherapy 38(1): 7-12. Available at: http://www.ncbi.nlm.nih.gov/pubmed/8141583 (accessed 28 November 2018).

Brown AE, Jones DE, Walker M, et al. (2015) Abnormalities of AMPK activation and glucose uptake in cultured skeletal muscle cells from individuals with chronic fatigue syndrome. PLoS ONE 10(4): e0122982. DOI: 10.1371/journal.pone.0122982.

Buchwald D, Herrell R, Ashton S, et al. (2001) A twin study of chronic fatigue. Psychosomatic medicine 63(6): 936-43. Available at: http://www.ncbi.nlm.nih.gov/pubmed/11719632 (accessed 3 July 2019).

Cairns R and Hotopf M (2005) A systematic review describing the prognosis of chronic fatigue syndrome. Occup Med (Lond) 55(1): 20-31. Available at:

http://www.ncbi.nlm.nih.gov/entrez/query.fcgi? $\mathrm{cmd}=$ Retrieve \&db=PubMed\&dopt=Citation\&list_u ids $=15699087$.

Cambras T, Castro-Marrero J, Cleofé Zaragoza M, et al. (2018) Circadian rhythm abnormalities and autonomic dysfunction in patients with Chronic Fatigue Syndrome/Myalgic Encephalomyelitis. DOI: 10.1371/journal.pone.0198106.

Carruthers BM, Jain AK, DeMeirleir KL, et al. (2003) Myalgic Encephalomyelitis/Chronic Fatigue 
Syndrome: Clinical Working Case Definition, Diagnostic and Treatment Protocols. Journal of Chronic Fatigue Syndrome 11(1): 7-36. DOI: 10.1300/J092v11n01.

Centers for Disease Control and Prevention (2006) Genetic And Environmental Factors Impact Chronic Fatigue Syndrome Patients. Available at: www.sciencedaily.com/releases/2006/04/060421112146.htm.

CFS/ME Working Group (2002) Report to the Chief Medical Officer of an Independent Working Group. London, UK. Available at: https://www.meassociation.org.uk/wp-content/uploads/CMO-Report2002.pdf (accessed 17 January 2019).

Chaudhuri A and Behan PO (2000) Fatigue and basal ganglia. Journal of the Neurological Sciences 179(1-2): 34-42. DOI: 10.1016/S0022-510X(00)00411-1.

Chaudhuri A, Watson WS, Pearn J, et al. (2000) The symptoms of chronic fatigue syndrome are related to abnormal ion channel function. Medical Hypotheses 54(1): 59-63. DOI:

10.1054/mehy.1998.0822.

Cho HJ, Menezes PR, Hotopf M, et al. (2009) Comparative epidemiology of chronic fatigue syndrome in Brazilian and British primary care: prevalence and recognition. Br J Psychiatry 194(2): 117-122. DOI: 10.1192/bjp.bp.108.051813.

Chu L, Valencia IJ, Garvert DW, et al. (2019) Onset Patterns and Course of Myalgic Encephalomyelitis/Chronic Fatigue Syndrome. Frontiers in Pediatrics 7. Frontiers: 12. DOI: 10.3389/fped.2019.00012.

Clark MR, Katon W, Russo J, et al. (1995) Chronic fatigue: Risk factors for symptom persistence in a 2 1/2-year follow-up study. The American Journal of Medicine 98(2). Elsevier: 187-195. DOI: 10.1016/S0002-9343(99)80403-3.

Cliff JM, King EC, Lee J-S, et al. (2019) Cellular Immune Function in Myalgic Encephalomyelitis/Chronic Fatigue Syndrome (ME/CFS). Frontiers in Immunology www.frontiersin.org 1: 796. DOI: 10.3389/fimmu.2019.00796.

Dahlgren G and Whitehead M (1991) Policies and strategies to promote social equity in health Background document to WHO - Strategy paper. Strategy paper for Europe. Stockholm, Sweden.

De Stavola BL, Nitsch D, dos Santos Silva I, et al. (2006) Statistical Issues in Life Course Epidemiology. American Journal of Epidemiology 163(1). Oxford University Press: 84-96. DOI: 10.1093/aje/kwj003.

Dubin AE and Patapoutian A (2010) Nociceptors: the sensors of the pain pathway. Journal of Clinical Investigation 120(11). American Society for Clinical Investigation: 3760-3772. DOI: $10.1172 / \mathrm{JCI} 42843$.

Dubois B, Hampel H, Feldman HH, et al. (2016) Preclinical Alzheimer's disease: Definition, natural history, and diagnostic criteria. Alzheimer's \& Dementia 12(3). Elsevier: 292-323. DOI: 10.1016/J.JALZ.2016.02.002.

Edwards JCW, McGrath S, Baldwin A, et al. (2016) The biological challenge of myalgic encephalomyelitis/chronic fatigue syndrome: a solvable problem. Fatigue : biomedicine, health \& behavior 4(2). Taylor \& Francis: 63-69. DOI: 10.1080/21641846.2016.1160598.

Esfandyarpour R, Kashi A, Nemat-Gorgani M, et al. (2019) A nanoelectronics-blood-based diagnostic biomarker for myalgic encephalomyelitis/chronic fatigue syndrome (ME/CFS). Proceedings of the National Academy of Sciences of the United States of America 116(21). National Academy of Sciences: 10250-10257. DOI: 10.1073/pnas.1901274116.

Esterov D and Greenwald B (2017) Autonomic Dysfunction after Mild Traumatic Brain Injury. Brain Sciences 7(12): 100. DOI: 10.3390/brainsci7080100.

Fennell PA (1995) The Four Progressive Stages of the CFS Experience: A Coping Tool for Patients. 
Journal of Chronic Fatigue Syndromeme 1(3-4): 69-79. DOI: 10.1300/J092v01n03_11.

Friedberg F, Dechene L, McKenzie MJ, et al. (2000) Symptom patterns in long-duration chronic fatigue syndrome. Journal of Psychosomatic Research 48(1). Elsevier: 59-68. DOI: 10.1016/S00223999(99)00077-X.

Fukuda K, Straus SE, Hickie I, et al. (1994) The chronic fatigue syndrome: a comprehensive approach to its definition and study. International Chronic Fatigue Syndrome Study Group. Annals of internal medicine 121(12): 953-959. DOI: 10.7326/0003-4819-121-12-199412150-00009.

Germain A, Ruppert D, Levine SM, et al. (2017) Metabolic profiling of a myalgic encephalomyelitis/chronic fatigue syndrome discovery cohort reveals disturbances in fatty acid and lipid metabolism. Molecular bioSystems 13(2). NIH Public Access: 371-379. DOI: 10.1039/c6mb00600k.

Germain A, Ruppert D, Levine S, et al. (2018) Prospective Biomarkers from Plasma Metabolomics of Myalgic Encephalomyelitis/Chronic Fatigue Syndrome Implicate Redox Imbalance in Disease Symptomatology. Metabolites 8(4). Multidisciplinary Digital Publishing Institute: 90. DOI: 10.3390/metabo8040090.

Glassford JAG (2017) The Neuroinflammatory Etiopathology of Myalgic Encephalomyelitis/Chronic Fatigue Syndrome (ME/CFS). Frontiers in Physiology 8(88). Frontiers: 1-9. DOI: 10.3389/fphys.2017.00088.

Haendel MA, Chute CG and Robinson PN (2018) Classification, Ontology, and Precision Medicine. New England Journal of Medicine 379(15): 1452-1462. DOI: 10.1056/NEJMra1615014.

Hatziagelaki E, Adamaki M, Tsilioni I, et al. (2018) Myalgic Encephalomyelitis/Chronic Fatigue Syndrome-Metabolic Disease or Disturbed Homeostasis due to Focal Inflammation in the Hypothalamus? Journal of Pharmacology and Experimental Therapeutics. DOI: 10.1124/jpet.118.250845.

Herman JP, Mcklveen JM, Ghosal S, et al. (2016) Regulation of the hypothalamic-pituitaryadrenocortical stress response. Compr Physiol 6(2): 603-21. DOI: 10.1002/cphy.c150015.

Higginbottom K (2018) The Price Of Presenteeism. Available at: https://www.forbes.com/sites/karenhigginbottom/2018/04/20/the-price-of-presenteeism-2/.

Hornig M, Montoya JG, Klimas NG, et al. (2015) Distinct plasma immune signatures in ME/CFS are present early in the course of illness. Science Advances 1(1): 1-10. DOI: 10.1126/sciadv.1400121.

Hotopf M, Noah N and Wessely S (1996) Chronic fatigue and minor psychiatric morbidity after viral meningitis: a controlled study. Journal of Neurology, Neurosurgery, and Psychiatry 60(5): 504509. DOI: 10.1136/jnnp.60.5.504.

$\mathrm{Hu} \mathrm{H}$ and Baines C (2018) Recent insights into 3 underrecognized conditions: Myalgic encephalomyelitis-chronic fatigue syndrome, fibromyalgia, and environmental sensitivitiesmultiple chemical sensitivity. Canadian family physician Medecin de famille canadien 64(6): 413415. Available at: http://www.ncbi.nlm.nih.gov/pubmed/29898928 (accessed 31 July 2019).

Institute of Medicine (IOM) (2015) Beyond Myalgic Encephalomyelitis/Chronic Fatigue Syndrome: Redefining an Illness. Washington, DC: The National Academies Press. Available at: http://www.nap.edu/catalog.php?record_id=19012 (accessed 9 January 2019).

Jamoulle M (2015) Quaternary prevention, an answer of family doctors to overmedicalization. Int J Health Policy Manag 4(2): 61-64. DOI: 10.15171/ijhpm.2015.24.

Janal MN, Ciccone DS and Natelson BH (2006) Sub-typing CFS patients on the basis of 'minor' symptoms. Biol Psychol 73(2). 2006/02/14.: 124-31. Epub 2006 Feb 10. DOI:

10.1016/j.biopsycho.2006.01.003.

Jason L, Muldowney K and Torres-Harding S (2008) The Energy Envelope Theory and myalgic 
encephalomyelitis/chronic fatigue syndrome. AAOHN journal : official journal of the American Association of Occupational Health Nurses 56(5): 189-95. Available at: http://www.ncbi.nlm.nih.gov/pubmed/18578185 (accessed 25 June 2019).

Jason LA, Fennell PA, Klein S, et al. (1999) An Investigation of the Different Phases of the CFS illness. Journal of Chronic Fatigue Syndrome 5(3-4): 35-54. DOI: 10.1300/J092v05n03_03.

Jason LA, Corradi K, Torres-Harding S, et al. (2005) Chronic fatigue syndrome: the need for subtypes. Neuropsychol Rev 15(1). 2005/06/03.: 29-58. Available at:

http://www.ncbi.nlm.nih.gov/entrez/query.fcgi? $\mathrm{cmd}=$ Retrieve\&db=PubMed\&dopt=Citation\&list_u ids $=15929497$.

Jewell NP (2016) Natural history of diseases: Statistical designs and issues. Clinical pharmacology and therapeutics 100(4). NIH Public Access: 353-61. DOI: 10.1002/cpt.423.

Joyce J, Hotopf M and Wessely S (1997) The prognosis of chronic fatigue and chronic fatigue syndrome: a systematic review. QJM : monthly journal of the Association of Physicians 90: 22333. Available at: http://www.ncbi.nlm.nih.gov/pubmed/9093600 (accessed 15 January 2019).

Kalogeris T, Baines CP, Krenz M, et al. (2012) Cell biology of ischemia/reperfusion injury. International review of cell and molecular biology 298. NIH Public Access: 229-317. DOI: 10.1016/B978-0-12-394309-5.00006-7.

Katz BZ, Shiraishi Y, Mears CJ, et al. (2009) Chronic Fatigue Syndrome Following Infectious Mononucleosis in Adolescents: A Prospective Cohort Study. Pediatrics 124(1). NIH Public Access: 189. DOI: 10.1542/PEDS.2008-1879.

Keel M and Trentz O (2005) Pathophysiology of polytrauma. Injury 36(6). Elsevier: 691-709. DOI: 10.1016/J.INJURY.2004.12.037.

Keller BA, Pryor JL and Giloteaux L (2014) Inability of myalgic encephalomyelitis/chronic fatigue syndrome patients to reproduce VO2peak indicates functional impairment. Journal of Translational Medicine. DOI: 10.1186/1479-5876-12-104.

Keller RH, Lane JL, Klimas N, et al. (1994) Association between HLA class II antigens and the chronic fatigue immune dysfunction syndrome. Clin Infect Dis 18 Suppl 1: S154-6. Available at: http://www.ncbi.nlm.nih.gov/pubmed/8148444.

Kerr JR, Burke B, Petty R, et al. (2008) Seven genomic subtypes of chronic fatigue syndrome/myalgic encephalomyelitis: a detailed analysis of gene networks and clinical phenotypes. J Clin Pathol 61(6): 730-739. DOI: 10.1136/jcp.2007.053553.

Klimas NG, Broderick G and Fletcher MA (2012) Biomarkers for chronic fatigue. Brain Behav Immun. Available at: http://www.ncbi.nlm.nih.gov/pubmed/22732129.

Komaroff AL (2019) Advances in Understanding the Pathophysiology of Chronic Fatigue Syndrome. JAMA. DOI: 10.1001/jama.2019.8312.

Kroenke K, Wood DR, Mangelsdorff AD, et al. (1988) Chronic Fatigue in Primary Care: Prevalence, patient characteristics, and outcome. JAMA 260(7). American Medical Association: 929. DOI: 10.1001/jama.1988.03410070057028.

Lacerda EM, Bowman EW, Cliff JM, et al. (2017) The UK ME/CFS Biobank for biomedical research on Myalgic Encephalomyelitis/Chronic Fatigue Syndrome (ME/CFS) and Multiple Sclerosis. Open Journal of Bioresources 4(1). 2017/12/19.: 4. DOI: 10.5334/ojb.28.

Last J (2014) A Dictionary of Epidemiology. Porta M (ed.). New York: Oxford University Press.

Levine PH, Jacobson S, Pocinki AG, et al. (1992) Clinical, Epidemiologic, and Virologic Studies in Four Clusters of the Chronic Fatigue Syndrome. Archives of Internal Medicine 152(8). American Medical Association: 1611. DOI: 10.1001/archinte.1992.00400200049009.

Levine PH, Snow PG, Ranum BA, et al. (1997) Epidemic Neuromyasthenia and Chronic Fatigue 
Syndrome in West Otago, New Zealand. Archives of Internal Medicine 157(7). American Medical Association: 750. DOI: 10.1001/archinte.1997.00440280064005.

Levy MM, Fink MP, Marshall JC, et al. (2003) 2001 SCCM/ESICM/ACCP/ATS/SIS International Sepsis Definitions Conference. Critical Care Medicine 31(4). Critical Care Medicine: 1250-1256. DOI: 10.1097/01.ccm.0000050454.01978.3b.

Lord JM, Midwinter MJ, Chen Y-F, et al. (2014) The systemic immune response to trauma: an overview of pathophysiology and treatment. The Lancet 384(9952). Elsevier: 1455-1465. DOI: 10.1016/S0140-6736(14)60687-5.

Low CA, Kalinski P and Bovbjerg DH (2015) Neurocognitive Impairment as One Facet of CancerRelated Sickness Behavior Symptoms. Journal of the National Cancer Institute 107(8): 176. DOI: 10.1093/jnci/djv176.

Maes M, Kubera M, Uytterhoeven M, et al. (2011) Increased plasma peroxides as a marker of oxidative stress in myalgic encephalomyelitis/chronic fatigue syndrome (ME/CFS). Medical Science Monitor: International Medical Journal of Experimental and Clinical Research 17(4). International Scientific Information, Inc.: 11-5. DOI: 10.12659/MSM.881699.

Marks DF (2017) Special issue on the PACE Trial. Journal of Health Psychology 22(9). SAGE PublicationsSage UK: London, England: 1103-1105. DOI: 10.1177/1359105317722370.

Martin L, Koczera P, Zechendorf E, et al. (2016) The Endothelial Glycocalyx: New Diagnostic and Therapeutic Approaches in Sepsis. BioMed Research International 2016(3758278). Hindawi Limited: 8. DOI: 10.1155/2016/3758278.

Martins C, Godycki-Cwirko M, Heleno B, et al. (2018) Quaternary prevention: reviewing the concept. The European journal of general practice 24(1). Taylor \& Francis: 106-111. DOI: 10.1080/13814788.2017.1422177.

McEwen BS (2005) Stressed or stressed out: What is the difference? Journal of Psychiatry and Neuroscience. Available at: https://www.ncbi.nlm.nih.gov/pmc/articles/PMC1197275/pdf/20050900s00002p315.pdf (accessed 30 October 2018).

Mello P, Gusmao-Flores D and Dellinger RP (2016) Sepsis. In: O’Donnell JM and Nácul FE (eds) Surgical Intensive Care Medicine. Third. Cham: Springer International Publishing, pp. 373-387.

Monro JA and Puri BK (2018) A Molecular Neurobiological Approach to Understanding the Aetiology of Chronic Fatigue Syndrome (Myalgic Encephalomyelitis or Systemic Exertion Intolerance Disease) with Treatment Implications. Molecular Neurobiology 55(9). Springer US: 7377-7388. DOI: $10.1007 / \mathrm{s} 12035-018-0928-9$.

Morris G, Berk M and Puri BK (2018) A Comparison of Neuroimaging Abnormalities in Multiple Sclerosis, Major Depression and Chronic Fatigue Syndrome (Myalgic Encephalomyelitis): is There a Common Cause? Molecular Neurobiology 55(4). Springer US: 3592-3609. DOI: 10.1007/s12035-017-0598-z.

Nacul L, Perreira F, Jacques G, et al. (1999) What do doctors and health workers of Northeast Brazil know and do about chronic fatigue syndrome? J Chronic Fatigue Syndrome 5: 89-90.

Nacul L, Lacerda EM, Kingdon CC, et al. (2017) How have selection bias and disease misclassification undermined the validity of myalgic encephalomyelitis/chronic fatigue syndrome studies? Journal of Health Psychology 1359105317. 2018/03/15.: 1-7. DOI: 10.1177/1359105317695803.

Nacul LC, Lacerda EM and Sakellariou D (2009) Is there an association between exposure to chemicals and chronic fatigue syndrome? Review of the evidence. Bulletin of IACFS/ME. Available at: http://www.iacfsme.org/BULLETINSPRING2009/tabid/363/Default.aspx.

Nacul LC, Lacerda EM, Pheby D, et al. (2011) Prevalence of myalgic encephalomyelitis/chronic fatigue 
syndrome (ME/CFS) in three regions of England: a repeated cross-sectional study in primary care. BMC Med 9: 91. DOI: 10.1186/1741-7015-9-91.

Naess H, Nyland M, Hausken T, et al. (2012) Chronic fatigue syndrome after Giardia enteritis: clinical characteristics, disability and long-term sickness absence. BMC Gastroenterol. 12:13.(doi). 2012/02/10.: 13. DOI: 10.1186/1471-230X-12-13.

Nagy-Szakal D, Barupal DK, Lee B, et al. (2018) Insights into myalgic encephalomyelitis/chronic fatigue syndrome phenotypes through comprehensive metabolomics. Scientific Reports 8(1). Nature Publishing Group: 10056. DOI: 10.1038/s41598-018-28477-9.

Natelson BH (2008) Your Symptoms Are Real. Hoboken: John Wiley \& Sons, Inc.

Natelson BH, Weaver SA, Tseng CL, et al. (2005) Spinal fluid abnormalities in patients with chronic fatigue syndrome. Clin Diagn Lab Immunol 12(1): 52-55. Available at:

http://www.ncbi.nlm.nih.gov/entrez/query.fcgi? $\mathrm{cmd}=$ Retrieve $\& d b=P u b M e d \& d o p t=C i t a t i o n \& l i s t \_u$ ids $=15642984$.

National Institute of Clinical Excellence (2007) Chronic fatigue syndrome/myalgic encephalomyelitis (or encephalopathy): Diagnosis and management of CFS/ME in adults and children. London. Available at: https://www.nice.org.uk/guidance/cg53.

Naviaux RK, Naviaux JC, Li K, et al. (2016) Metabolic features of chronic fatigue syndrome. Proceedings of the National Academy of Sciences of the USA 113(37): 5472-5480. DOI: 10.1073/pnas.1607571113.

Newton DJ, Kennedy G, Chan KKF, et al. (2012) Large and small artery endothelial dysfunction in chronic fatigue syndrome is. DOI: 10.1016/j.ijcard.2011.10.030.

Nisenbaum R, Jones JF, Unger ER, et al. (2003) A population-based study of the clinical course of chronic fatigue syndrome. Health and Quality of Life Outcomes 1(1). BioMed Central: 49. DOI: 10.1186/1477-7525-1-49.

Njoku MG, Jason LA and Torres-Harding SR (2007) The prevalence of chronic fatigue syndrome in Nigeria. J Health Psychol 12(3). 2007/04/19.: 461-474. DOI: 12/3/461 [pii]10.1177/1359105307076233.

O’Donnell JM and Nacul FE (2016) Surgical Intensive Care Medicine. Third. O'Donnell JM and Nacul FE (eds). Basel: Springer International Publishing. DOI: 10.1007/978-3-319-19668-8.

Oosterwijck J Van, Marusic U, De Wandele I, et al. (2017) The Role of Autonomic Function in Exercise-induced Endogenous Analgesia: A Case-control Study in Myalgic Encephalomyelitis/Chronic Fatigue Syndrome and Healthy People. Pain physician 20(3): E389E399. Available at: http://www.ncbi.nlm.nih.gov/pubmed/28339438 (accessed 4 July 2019).

Pall ML (2000) Elevated Peroxynitrite as the Cause of Chronic Fatigue Syndrome. Journal of Chronic Fatigue Syndrome 7(4). Taylor \& Francis: 45-58. DOI: 10.1300/J092v07n04_05.

Pall ML (2003) Elevated nitric oxide/peroxynitrite theory of multiple chemical sensitivity: central role of N-methyl-D-aspartate receptors in the sensitivity mechanism. Environmental Health Perspectives 111(12): 1461-1464. DOI: 10.1289/ehp.5935.

Pool R, Gomez H and Kellum JA (2018) Mechanisms of Organ Dysfunction in Sepsis. Critical Care Clinics 34(1). Elsevier: 63-80. DOI: 10.1016/J.CCC.2017.08.003.

Prins JB, van der Meer JWM and Bleijenberg G (2006) Chronic fatigue syndrome. The Lancet 367(9507): 346-355. DOI: 10.1016/s0140-6736(06)68073-2.

Prüss H, Tedeschi A, Thiriot A, et al. (2017) Spinal cord injury-induced immunodeficiency is mediated by a sympathetic-neuroendocrine adrenal reflex. Nature Neuroscience 20(11): 1549-1559. DOI: 10.1038/nn.4643.

Rajeevan MS, Dimulescu I, Murray J, et al. (2015) Pathway-focused genetic evaluation of immune and 
inflammation related genes with chronic fatigue syndrome. Hum Immunol 76(8): 553-560. DOI: 10.1016/j.humimm.2015.06.014.

Ramsay A (1986) Postviral Fatigue Syndrome-The Saga of Royal Free Disease. 1st ed. London, UK: Gower Medical Publishing Limited. Available at: https://www.meassociation.org.uk/shop/books/saga-of-royal-free-disease/ (accessed 30 October 2018).

Rathour S, Kumar S, Hadda V, et al. (2015) PIRO concept: staging of sepsis. Journal of postgraduate medicine 61(4). Wolters Kluwer -- Medknow Publications: 235-42. DOI: 10.4103/00223859.166511 .

Reynolds GK, Lewis DP, Richardson AM, et al. (2014) Comorbidity of postural orthostatic tachycardia syndrome and chronic fatigue syndrome in an Australian cohort. Journal of Internal Medicine 275(4): 409-417. DOI: 10.1111/joim.12161.

Rosenblatt MS and Delmonico TR (2016) Trauma. In: O’Donnell JM and Nácul FE (eds) Surgical Intensive Care Medicine. Third. Cham: Springer International Publishing, pp. 599-610. DOI: 10.1007/978-3-319-19668-8_44.

Rowe PC, Fontaine KR and Violand RL (2013) Neuromuscular strain as a contributor to cognitive and other symptoms in chronic fatigue syndrome: hypothesis and conceptual model. Frontiers in Physiology 4. Frontiers: 115. DOI: 10.3389/fphys.2013.00115.

Rowe PC, Fontaine KR, Lauver M, et al. (2016) Neuromuscular Strain Increases Symptom Intensity in Chronic Fatigue Syndrome. PLoS ONE 11(7): e0159386. DOI: 10.1371/journal.pone.0159386.

Rowe PC, Underhill RA, Friedman KJ, et al. (2017) Myalgic Encephalomyelitis/ Chronic Fatigue Syndrome Diagnosis and Management in Young People: A Primer. Front. Pediatr 5: 121. DOI: 10.3389/fped.2017.00121.

Russell A, Hepgul N, Nikkheslat N, et al. (2019) Persistent fatigue induced by interferon-alpha: a novel, inflammation-based, proxy model of chronic fatigue syndrome. Psychoneuroendocrinology 100. Pergamon: 276-285. DOI: 10.1016/J.PSYNEUEN.2018.11.032.

Russell JA, Rush B and Boyd J (2018) Pathophysiology of Septic Shock. Critical Care Clinics 34(1). Elsevier: 43-61. DOI: 10.1016/j.ccc.2017.08.005.

Russell L, Broderick G, Taylor R, et al. (2016) Illness progression in chronic fatigue syndrome: a shifting immune baseline. BMC Immunology 17(1). BioMed Central: 3. DOI: 10.1186/s12865-0160142-3.

Rutherford G, Manning P and Newton JL (2016) Understanding Muscle Dysfunction in Chronic Fatigue Syndrome. J Aging Res 2016. Hindawi Publishing Corporation: 2497348. DOI: $10.1155 / 2016 / 2497348$.

Schlauch KA, Khaiboullina SF, De Meirleir KL, et al. (2016) Genome-wide association analysis identifies genetic variations in subjects with myalgic encephalomyelitis/chronic fatigue syndrome. Transl Psychiatry 6: e730. DOI: 10.1038/tp.2015.208.

Schutzer SE, Angel TE, Liu T, et al. (2011) Distinct Cerebrospinal Fluid Proteomes Differentiate PostTreatment Lyme Disease from Chronic Fatigue Syndrome. Gendelman H (ed.) PLoS ONE 6(2). Public Library of Science: e17287. DOI: 10.1371/journal.pone.0017287.

Schweitzer MM (1999) WECAN Testimony to the Chronic Fatigue Syndrome Coordinating Committee of DHHS. Available at: http://www.cfids-me.org/cfscc/cfsccs98.html (accessed 4 January 2019).

Seet RCS, Quek AML and Lim ECH (2007) Post-infectious fatigue syndrome in dengue infection. Journal of Clinical Virology 38(1). Elsevier: 1-6. DOI: 10.1016/J.JCV.2006.10.011.

Shepherd C and Chaudhuri A (2019) ME/CFS/PVFS: An Exploration of the Key Clinical Issues. 11th ed. Gawco: The ME Association. 
Singer M (2014) The role of mitochondrial dysfunction in sepsis-induced multi-organ failure. Virulence 5(1): 66-72. DOI: 10.4161/viru.26907.

Smith AK, White PD, Aslakson E, et al. (2006) Polymorphisms in genes regulating the HPA axis associated with empirically delineated classes of unexplained chronic fatigue. Pharmacogenomics 7(3). Future Medicine Ltd London, UK: 387-394. DOI: 10.2217/14622416.7.3.387.

Smith AK, Fang H, Whistler T, et al. (2011) Convergent genomic studies identify association of GRIK2 and NPAS2 with chronic fatigue syndrome. Neuropsychobiology 64(4): 183-194. DOI: $10.1159 / 000326692$.

Smith J, Fritz EL, Kerr JR, et al. (2005) Association of chronic fatigue syndrome with human leucocyte antigen class II alleles. J Clin Pathol 58(8): 860-863. DOI: 10.1136/jcp.2004.022681.

Stewart WF, Ricci JA, Chee E, et al. (2003) Lost Productive Time and Cost Due to Common Pain Conditions in the US Workforce. JAMA 290(18). American Medical Association: 2443. DOI: 10.1001/jama.290.18.2443.

Tomas C, Newton J and Watson S (2013) A review of hypothalamic-pituitary-adrenal axis function in chronic fatigue syndrome. ISRN neuroscience 2013. Hindawi Limited: 784520. DOI: $10.1155 / 2013 / 784520$.

Twisk F (2018) Myalgic Encephalomyelitis (ME) or What? An Operational Definition. Diagnostics 8(3): 64. DOI: 10.3390/diagnostics8030064.

van Campen C (Linda) MC, Rowe PC and Visser FC (2018) Blood Volume Status in ME/CFS Correlates With the Presence or Absence of Orthostatic Symptoms: Preliminary Results. Frontiers in Pediatrics 6. Frontiers: 352. DOI: 10.3389/fped.2018.00352.

VanElzakker MB, Brumfield SA and Lara Mejia PS (2019) Neuroinflammation and cytokines in myalgic encephalomyelitis/chronic fatigue syndrome (ME/CFS): A critical review of research methods. Frontiers in Neurology. DOI: 10.3389/fneur.2018.01033.

Vermeulen RC and Vermeulen van Eck IW (2014) Decreased oxygen extraction during cardiopulmonary exercise test in patients with chronic fatigue syndrome. Journal of Translational Medicine 12(1). BioMed Central: 20. DOI: 10.1186/1479-5876-12-20.

Walsh CM, Zainal NZ, Middleton SJ, et al. (2001) A family history study of chronic fatigue syndrome. Psychiatric genetics 11(3): 123-8. Available at: http://www.ncbi.nlm.nih.gov/pubmed/11702053 (accessed 3 July 2019).

White PD, Thomas JM, Amess J, et al. (1998) Incidence, risk and prognosis of acute and chronic fatigue syndromes and psychiatric disorders after glandular fever. British Journal of Psychiatry 173(6). 1999/02/02. Cambridge University Press: 475-481. DOI: 10.1192/bjp.173.6.475.

White PD, Goldsmith KA, Johnson AL, et al. (2011) Comparison of adaptive pacing therapy, cognitive behaviour therapy, graded exercise therapy, and specialist medical care for chronic fatigue syndrome (PACE): a randomised trial. Lancet 377(9768). Elsevier Ltd: 823-836. DOI: 10.1016/S0140-6736(11)60096-2.

Wilshire C, Kindlon T, Matthees A, et al. (2017) Can patients with chronic fatigue syndrome really recover after graded exercise or cognitive behavioural therapy? A critical commentary and preliminary re-analysis of the PACE trial. Fatigue: Biomedicine, Health \& Behavior 5(1). Taylor \& Francis: 43-56. DOI: 10.1080/21641846.2017.1259724.

Wrona D (2006) Neural-immune interactions: An integrative view of the bidirectional relationship between the brain and immune systems. Journal of Neuroimmunology 172(1-2): 38-58. DOI: 10.1016/j.jneuroim.2005.10.017.

Zhang L, Gough J, Christmas D, et al. (2010) Microbial infections in eight genomic subtypes of chronic fatigue syndrome/myalgic encephalomyelitis. J Clin Pathol 63(2): 156-164. Available at: 
http://www.ncbi.nlm.nih.gov/pubmed/19955554. 